\title{
Understanding how student nurses experience morally distressing situations: Caring for patients with different values and beliefs in the clinical environment
}

\author{
Mary J o C Stanley ${ }^{1}$, Nancy J. Matchett ${ }^{2}$ \\ 1. University of Colorado at Colorado Springs, Colorado Springs, United States. 2. University of Northern Colorado, \\ Greeley, United States. \\ Correspondence: Mary J o C Stanley. Address: University of Colorado, Colorado Springs, United States. \\ Email: joey-stanley@hotmail.com
}

Received: July 8, 2014

Accepted: August 5, 2014

Online Published: August 24, 2014

DOI : $10.5430 /$ jnep.v4n10p133

URL: http://dx.doi.org/10.5430/jnep.v4n10p133

\section{Abstract}

Introduction/Background: Moral distress and related concepts surrounding morality and ethical decision-making have been given much attention in nursing. Despite the general consensus that moral distress is an affective response to being unable to act morally, the literature attests to the need for increased clarity regarding theoretical and conceptual constructs used to describe precisely what the experience of moral distress involves. The purpose of this study is to understand how student nurses experience morally distressing situations when caring for patients with different values and beliefs than their own in the clinical environment

Methods: This study is based on secondary analysis of participant data. The stories of eight student nurses who completed the original study were reviewed following Yin’s multiple-case study design.

Results: Findings suggest there is a subtle form of moral distress that has been under appreciated in the literature and differs from Jameton's classic definition. While traditional institutional triggers to moral distress are pervasive, personal conflict as a result of differing value systems may be a moral challenge faced by nursing students working with culturally diverse patients.

Conclusion: Ethics education is needed in nursing school to reduce moral distress in the clinical environment. Nursing students need opportunities to develop moral reasoning skills in addition to their clinical skills. A philosophical approach to ethics education may be needed to prevent and alleviate moral distress.

\section{Key words}

Moral distress, Cutural diversity, Value systems, Ethics education

\section{I ntroduction and background}

Moral distress and related concepts surrounding morality and ethical decision-making have been given much attention in nursing. Andrew Jameton ${ }^{[1]}$ first defined moral distress as occurring “....when one knows the right thing to do, but 
institutional constraints make it nearly impossible to pursue the right course of action”. The concept of moral distress has been linked to the stressful work environment of nursing where nurses are conflicted and at odds with institutional practices and authoritarian structures. The negative effects of moral distress in the workplace and the implications for work satisfaction, nurse recruitment and retention, and quality patient care have been well documented ${ }^{[2-6]}$. More recently, moral distress has been used to describe a wide range of responses to morally challenging patient situations encountered by nurses in the course of providing patient care. Recent research suggests that Jameton's ${ }^{[1]}$ original definition does not capture the personal experiences of nurses who feel morally constrained or morally challenged ${ }^{[2,5-9]}$.

The literature attests to the need for increased clarity regarding theoretical and conceptual constructs that define moral distress, and suggest a more philosophical perspective may be needed to understand and resolve morally distressing situations in the clinical environment ${ }^{[2,5,6,8]}$. Pauly, Varcoe and Storch ${ }^{[2]}$ propose inclusion of a philosophical perspective in health care education that informs moral decision making processes and emotional response; attention must be shifted from the ethical dilemma to application of an ethical framework that informs everyday practice ${ }^{[2]}$. Similarly, Nathaniel ${ }^{[5]}$ highlights the practical and relational aspects of nursing and the potential conflicts between personal and professional values, using the term "situational bind" to describe the experiences of nurses whose core beliefs come into irreconcilable conflict with differing value systems ${ }^{[5]}$. In a study of end-of-life decision making, Repenshek ${ }^{[6]}$ found that moral distress arises from nurses' discomfort with moral subjectivity. The fact that patients may value end-of-life outcomes differently than nurses can create tension within nurses' role morality ${ }^{[6]}$.

Other studies show that morally challenging situations may threaten nurses' sense of identity; hence the development of coping strategies that preserve nurses' moral integrity is critical ${ }^{[4]}$. When moral or ethical situations create internal conflict, the perceived challenge to core beliefs can produce personal tension causing physical and emotional responses when unresolved ${ }^{[5]}$. This "unrelieved distress" may jeopardize a nurse's sense of self-worth ${ }^{[10]}$. The development of a more philosophical outlook toward conflict, and increased ethical deliberation skills may be needed in nursing to prevent and alleviate moral distress in the clinical environment.

This study adds to the literature by attempting to understand how student nurses experience morally distressing situations when caring for patients with values and beliefs different from their own in the clinical environment. The present study is an attempt to explain findings from one co-author's dissertation work ${ }^{[11]}$. The original dissertation research sought to understand the meaning of student nurses' experiences of cultural diversity as experienced in their clinical environments in nursing school. Outcomes from the study indicated that nursing students experienced significant moral challenges when caring for culturally diverse individuals in their undergraduate clinical settings in nursing school. Since the degree of moral challenge identified in the dissertation study was unexpected, further examination was warranted. Collaborative efforts between professors from nursing and philosophical ethics helped guide this study and established the research question: How do student nurses experience morally challenging situations when caring for culturally diverse individuals in the clinical setting? Further examination of the relationship between moral distress as described in the nursing literature and ethical decision-making as conceptualized within the discipline of philosophy may provide insight for future educational practices.

\section{Methodology}

This multiple-case study is based on secondary analysis of data ${ }^{[11]}$. Participants in the original study were nursing students from a baccalaureate nursing program in the Rocky Mountain region in the United States. Senior level student nurses successfully completing all nursing classes and clinical work, except their final preceptor experience, met inclusion criteria. A convenience sample of 12 senior level student nurses out of a possible 35 accessible students began the study; eight students completed the study. All participants were 21 years of age or older and agreed to participate through signed consent. The initial dissertation study and the secondary analysis of data were IRB approved. 
The secondary analysis of data reviewed both journals and interviews collected during the dissertation study; no additional data was collected. The original study followed Denzin's ${ }^{[12]}$ qualitative design where procedural rigor through member checking and peer review was done. For the current study, the stories of all eight student nurses who completed the original study were reviewed following Yin's ${ }^{[13]}$ five components of multiple-case study design: a) The research question- the research question emerged in an effort to understand the findings from this co-author's dissertation work ${ }^{[11]}$, b) The study's propositions- full immersion in the eight cases where moral challenges were identified, combined with a review of the literature on moral distress led to the study's propositions: the moral challenges identified by students differed from the traditional distress pattern identified by Jameton ${ }^{[1]}$, and the coping strategies used by student nurses were inadequate to alleviate the core problem ; c) The unit of analysis- senior undergraduate female nursing students define the case; evidence of their experience was gathered through retrospective review of interviews and journals from each participant. The boundaries of the case include 20 months of clinical time in medical-surgical, pediatric, obstetric, mental health, and community health settings within an undergraduate nursing baccalaureate program; d) Link the data to the propositionsthe eight case studies were extensively reviewed from a nursing and philosophical lens; cross-case analysis identified limited philosophical appreciation for the moral challenges experienced by the participants; e) Interpreting the findingsstudents' experiences of moral distress were identified as falling into one or more of three identified categories: those triggered by institutional obstacles that interfered with the duty to care (Jameton's ${ }^{[1]}$ definition of moral distress), those that emerged from conflict between student's personal moral values and the moral values of patients with different practices and beliefs (identified as value system conflict within this study), and the tendency for nursing students to ignore or discount their negative feelings related to morally challenging situations (identified in this study as emotional distancing).

\section{Results}

Findings from our research question "How do student nurses experience morally challenging situations when caring for culturally diverse individuals in the clinical setting?” indicate that nursing students experienced morally challenging situations in the clinical environment. All nursing students' experienced institutional obstacles that prevented them from doing what they feel or believe to be morally right. In addition, many students experienced value system conflict where student nurses were confronted by differing practices and beliefs, and were morally conflicted regarding the right course of action. Outcomes from the study also identified that students relied on emotional distancing as a way of avoiding moral conflict. All participants were female and between the ages of 21 and 28. Six of the eight participants identified themselves as white and having previous experience working with culturally diverse populations. Pseudonyms were used to present the research findings.

All eight participants identified morally challenging situations early on in their clinical rotations. Many of their stories support Jameton's ${ }^{[1]}$ previous work regarding moral distress since students did identify moral challenges related to institutional obstacles during their clinical experiences in nursing school. For example, one student nurse felt that her clinical observations were overlooked by doctors and floor nurses in a way that compromised care, and another was disturbed by a lack of communication between a non-English speaking patient and hospital staff. In addition, students described their discomfort when more experienced nurses stereotyped patients. Student nurses acknowledged that these behaviors were in tension with their duty to care:

"He only spoke Albanian, and so consequently, he was very confused about why he was being held in a hospital bed, why all these tubes were being prodded and poked into him, and why he wasn't going home. No one had explained to him the development in his situation, what the findings revealed, why he was being kept in a hospital, and what the plan of care was. Frankly, I couldn't imagine being in this patient's situation. It seems frightening enough to be sick, but to be sick and have no way to communicate needs, desires, or questions would leave me deathly frightened. He was in urgent need of care, no doubt, and very sick, but first I wanted him to understand what his condition was, what our plan of care was, and get him oriented to the situation.” (Jaclyn) 
"He was clearly withdrawing. He was clearly hallucinating. And then - the doctor was like I'm not going to write it. I'm not going to write it. So it was really I felt unethical to make this man suffer because of his addiction and like you don't want to write an order I feel isn't good enough. We just kept trying to get something done and it wouldn't happen. Like this shouldn't be happening. And it was really, really frustrating that they weren't taking care of his needs better.” (Amanda)

"So- and things like Hispanic patients not wanting epidurals. I worked with a lot of Hispanic patients that wanted them. So just assuming that they don't is an error.” (Mackenzie)

These students were confronted by behaviors and actions that were in conflict with their duty to care and the central ethical tenets of their profession. While tension in medical practice is not new, strategies for coping and dealing with these behaviors may be limited for student nurses.

\subsection{Value system conflict}

Institutional obstacles were not the only moral challenges experienced by student nurses. When asked to care for patients with different values and practices, many of the students began to question their understanding of "the right thing to do". For example, one student struggled to provide equal care to her patients; one of the student's patients was a methamphetamine dealer who had injured himself while the other patient suffered from disease complications. This student understood and expressed that she had a general duty to care equally for all patients, however, time constraints made it difficult for her to care effectively for both patients and she found herself wondering whether the second patient deserved more of her time:

"I guess the first time it really hit me was um I had a patient to take care of that was a drug dealer and had been cooking meth and he got burned. Blew his house up and got burned very badly. And um there was a second patient next door that I was supposed to be taking care of as well and he was unable to eat but needed help with eating and needed help with all sorts of things. And my time was very split between the two. And it was hard because there was a really big part of me that was like this other patient who is so deserving of my time and really just needs somebody there and he deserves it you know and I'm angry because there's this other patient who made a terrible choice and, as far as my personal opinion, and the lifestyle he led and - I'm a mom and thinking about my kid getting that stuff. Like it's - it really was a difficult situation for me and I cried thinking about what was right versus what I was feeling.” (Brittany)

Two participants struggled with patients who treated them, and all women, unequally. Both of these student nurses' commitments to equality between the sexes were in direct opposition to their patients' expressions of male dominance. The students acknowledged they had an obligation to provide the male patient with equal care, but this moral commitment was in tension with each student's feelings that the male patient's behavior toward women was reprehensible:

"And it was - it was really interesting to see - like getting around cultural barriers like one of my clients his wife - he was in the hospital but his wife did everything for him but she had no say in what she was doing. If that makes sense. It was more like a servant-master relationship but she was really okay with it. It was just part of their culture. And he - he would be like I need this now and she would be like okay I will go get it. And it wasn't - it wasn't like a loving relationship. It was a loving relationship but it wasn't like husband and wife like I've experienced it. It was more like this is my house, this is what we are doing, you need to do this for me because I am the master of this house...it was more like what he wanted went. It was - at first it was really overwhelming. Like my - my own beliefs like when he was talking to his wife in like more of a servant manner I was like no because my - my relationships are based on like equal trust and equal like rights and responsibilities and things like that." (Amanda) 
"For one example I worked with a younger male. I'm not sure exactly what specific culture it was but it was where more the men were more glorified and the women kind of did stuff for the guys. And so he had a broken arm and he wouldn't use the urinal by himself and would force the female nurses to help him. So learning how to handle that and how to respond knowing the culture and that's not necessarily because he is a rude person but it is the culture and it's what he is used to. So learning how to respond to that I think was the biggest thing I had to learn.” (Summer)

Another student struggled with values and cultural practices that were counter to her experiences, struggling to make sense of a value system “crazily different” from her own:

"One story that definitely stands out as trying for me and a huge learning experience in culture competence would have been during my rotation uh dealing with a particular woman who was first of all married and was thirteen and was pregnant with her second child, obviously all a result of the culture beliefs of that culture. Crazily different than my own, and, furthermore, dealing with the fact that she was fully circumcised and stitched up and now pregnant and in impending labor. And it was very hard um to negotiate through the process. I mean everything had to be negotiated. You had to get the husband's permission to hang Pitosin or to even just check her temperature. I mean to do every tiny little thing. And it was also hard - I think one of the biggest challenges I had to consciously overcome was I saw this thirteen year old and I almost instinctively just treated her like a child because to me she was one. But she was a woman giving birth who had accomplished more from motherhood and womanhood standpoint than I had even begun to think about in my own life.” (Jaclyn) ${ }^{[14]}$

Students also struggled with value system conflicts stemming from different religious convictions regarding life and death. One student's intent was to provide information the patient needed to make an informed decision, yet she was uncomfortable with the decision the patient made:

“We actually just had a Jehovah's Witness patient and she needed preferably a heart transplant but of course that was a big no. And they said well you know a pace maker would really also benefit you but we would have to give you some blood products. Well at first they didn’t use blood products. She needed platelets and she didn't understand what platelets were and was like, "Yeah I think I'll do that." because she was talking to the doctor about that. Then later one of the nurses went back in and told her. "You know platelets are a blood product right?" And she was like, "No. I can't do that then." So it is kind of interesting to see how she responded because at first she was like, "Oh yeah that would be really great if I could have that intervention to prolong my life." And then once she found out she was like, "No I can't do that. I need to stick to my beliefs." Sometimes it's hard as a medical professional to look at somebody and see that there is an intervention that we could do to help prolong their life or decrease they pain or whatever.” (Kiely)

Stories suggest that the distress felt by nursing students was not always the result of external obstacles or institutionally imposed constraints on nursing care, but, rather, occurred when nurses were confronted by differing value systems embedded in cultural practices unfamiliar or counter to the nurse's own values.

\subsection{Emotional distancing}

In several cases, students demonstrated limited coping strategies, especially in cases where the distress was triggered by a value system conflict. Stories indicate that nursing students relied on a strategy of distancing themselves from the difficult emotions, rather than attempting to resolve their inner conflict:

"And so like watching him boss her around like really upset me at first. So I tried to move past it. I wrote it down and then I - I wrote down like I'm not happy with this and then I threw it away because it kind of cleanses those bad feelings.” (Amanda) 
Amanda was confronted with a value belief different than her own. The male patient she cared for portrayed gender discrimination toward females; this was in tension with Amanda's personal beliefs and she struggled with her duty to provide care to this patient. Writing the negative feelings down and then throwing the paper away was a cathartic action that allowed Amanda to continue to provide care, yet did not allow her as a nurse and individual to process the differing value systems.

"Well when I first got a report on my patient and was taking care of him, I was angry. I took - I took a good twenty minutes um taking care of some of my other responsibilities before I even went into the room because I really felt I needed to accept the situation. I had taken on the responsibility by accepting you know the report and that the patient was going to be my responsibility. But I really felt that I needed to collect myself before going in there. And um that was probably the first initial stage of having to just deal with it. I feel like I did okay through my shift. I mean I feel like I worked really hard to treat him the same way that I was treating my other patient. And smiling and being friendly and you know wanting him to know that I was there and that I cared. And I am just kind of reflected on what I needed to do to get through that day. At that moment it was putting my feelings aside. Deal with this emotional later. Because right now this patient is my responsibility to take care of, and I did that.” (Brittany)

Brittany shares her conflict in caring for a methamphetamine dealer and the need to provide equal care. She describes acceptance of the situation so she can "get through the day" by allowing her own feeling to be "put aside" in order to provide care. Rather than attempting to resolve the value conflict, she is distancing herself from the situation.

"And coming to put my culture's beliefs aside of what I thought it meant to be a thirteen year old having a second kid. And my cultural beliefs aside about the man who would voluntarily want his wife to have all of that mutilation- And treat them - and - and take care of them and see them for what they were which was human beings.” (Jaclyn)

Jaclyn acknowledges that she must put her own values aside in order to care for this patient and her family. There is no pretense that she does not understand the beliefs of this patient, but her own morals dictate her value for life and the need to care for these people.

"I took care of a Jehovah's Witness and she had just gotten back from surgery and she had a lot of blood loss. And they don't do the human blood products and I was born and raised Catholic. So I don't know. It's kind of - that's kind of hard. I don't really want to say my morals were challenged but it was hard to see someone die because of they didn't want to receive the blood products. Because at the same time it was my religion that made me believe there is life after and there are greater things after you die and everything. So in one sense it was okay this is what she has accepted and she understands her risks and if she believes there is something better and she is not afraid and she wants to stand by her religion then good for her and I have respect for that. But then on the other hand you have the nurse caring side where you want to save and help as much as possible. But as far as moral challenges I don't feel I've really faced any hard moral questions yet. I'm sure I will. But I don't feel as if I've faced any really tough moral decisions.” (Summer) ${ }^{[14]}$

Summer acknowledges that she and her patient have different beliefs regarding religion, conceding that her nursing and personal ethics makes these values that much more difficult to accept. However, Summer has also distanced herself from the situation stating that she has not "faced any really tough moral decisions" and does not acknowledge this conflict in her own beliefs.

Participant stories suggest that many students rely on emotional distancing as a way of avoiding or simply ignoring the negative feelings associated with being confronted by differing value systems. Student nurses did acknowledge that they had to ignore or discount their negative feelings related to a situation in order to continue to perform effectively. While this 
strategy shows an admirable commitment to providing patient care, setting one's feelings aside is a way of avoiding, rather than resolving, the deeper value system conflict that the student is experiencing.

\subsection{Limitations}

Limitations of this study include the small sample size and the reliance on a single perspective; only eight student nurses' stories were reviewed and no inclusion of the staff, faculty, or patient perspective was provided.

\section{Discussion}

To answer the research question "How do student nurses experience morally challenging situations when caring for culturally diverse individuals in the clinical setting?” a secondary analysis of student nurses' stories regarding their experience of caring for culturally diverse populations was conducted. Examining the data through a philosophical and a nursing lens enabled researchers to identify a presentation of moral distress somewhat different than Jameton's definetion ${ }^{[1]}$. This new finding appears to be more internal; students' stories indicate a conflict between their personal moral values and the moral values of their patients; the source of the conflict is external (from the patient), yet the constraint is not. The nursing students in this case study were constrained by their own doubts about what they morally "ought to do", rather than by external forces that prevented them from acting on the desire to do what was right. The distress they experienced appeared to be as much cognitive/intellectual as affective/emotional.

This new finding may be closer to the "situational bind" described by Nathaniel ${ }^{[5]}$ or to what Repenshek ${ }^{[6]}$ describes as discomfort with the "subjectivity" of ethical decision making, than with literature which conceptualizes moral distress primarily as an affective response to external moral constraints. Rather than struggling with institutional obstacles, the nursing students found it difficult to determine whether or how to accept the patient's values as simply "different" without feeling that they were somehow sacrificing their own moral values. Students' struggles in this study are also similar to the distress described in Elpern's ${ }^{[15]}$ end-of-life study where nurse's subjectivity interferes with what is truly in the best interest of the patient. Value system conflict arises when nurses' own values are dissonant with the values of their patients. These feelings may be triggered by the uncertainty about how to provide care to patients whose values are radically different from their own.

Emotional distancing was the primary strategy students used to detach themselves from the internal struggle they may experience when conflicts arise between personal values and the values of the patient. While emotional distancing can provide an immediate release, it does not resolve the underlying cognitive conflict and does not enable the nurse to clearly articulate her own value commitments when caring for a patient. Working closely with patients who have very different value systems may trigger this kind of internal distress, and could create a tension between the nurse's role morality (the values that govern the nurse's understanding of herself as a nurse) and her broader personal morality (the values that determine what she herself might choose). Nurses who experience this internal conflict are torn between two competing value systems within their own thinking, and as Kelly ${ }^{[4]}$ suggests, this tension poses a threat to their moral integrity. Nursing students need coping strategies for dealing with morally distressing situations that assists them in acknowledging differences in beliefs.

These findings support existing literature suggesting that better ethics education may help reduce or resolve moral distress in nursing ${ }^{[2,16]}$. Epstein and Delgato ${ }^{[17]}$ state that current ethics education is inadequate and that "[v]alue clarification, communication skills, and an understanding of the system in which healthcare is delivered are the tools necessary to address conflicts between the internal and external environments”. These authors highlight the use of case studies as a way to assist students in identifying and discussing ethical principles. Nurse educators need opportunities to resolve value system conflicts within the clinical setting; learning to apply a more philosophical framework for resolving ethical conflicts may prepare students to encounter morally challenging situations in the clinical environment. It is crucial for 
nurses to develop skills in ethical deliberation that enable nurses to feel confident about their ability to rank and prioritize conflicting values.

\section{Conclusion}

Findings from this case study suggest that a philosophical approach to ethics education in nursing may be needed to prevent and alleviate morally distressing situations. Nurses need opportunities to develop more nuanced reasoning skills along with their clinical skills where course work is devoted to ethical development. Use of case studies can assist students in identifying and discussing ethical principles thereby developing the necessary skills to assist with ranking and prioritizing conflicting values. Nurse educators can assist students in developing ethical deliberation skills and coping strategies using experiential learning techniques, situated learning, and standardized patient simulations. While a complete critical outline of nursing ethics education is beyond the scope of this paper, further educational research is needed to identify best teaching strategies to assist students with morally distressing situations in the clinical environment.

\section{References}

[1] Jameton, A. Nursing practice: The ethical issues. Upper Saddle River, NJ: Prentice Hall. 1984. PMid:10265936

[2] Pauly, B., Varcoe, C., Storch, J. Framing the issues: Moral distress in health care. HEC Forum. 2012; 24: 1-11. PMid:22446885 http://dx.doi.org/10.1007/s10730-012-9176-y

[3] Huffman, D.M., \& Rittenmeyer, L. How professional nurses working in hospital environments experience moral distress: A systematic review. Critical Care Nursing Clinics of North America. 2012; 24(1): 91-100. PMid:22405714 http://dx.doi.org/10.1016/j.ccell.2012.01.004

[4] Kelly, B. Preserving moral integrity: A follow-up study with new graduate nurses. Journal of Advanced Nursing. 1998; 28(5): 1134-1145. http://dx.doi.org/10.1046/j.1365-2648.1998.00810.x

[5] Nathaniel, A. Moral reckoning in nursing. Western Journal of Nursing Research. 2006; 28: 419-438. PMid:16672631 http://dx.doi.org/10.1177/0193945905284727

[6] Repenshek, M. Moral distress: inability to act or discomfort with moral subjectivity? Nursing Ethics. 2009 ; $16(6)$ : 734-742. PMid:19889914 http://dx.doi.org/10.1177/0969733009342138

[7] McCarthy, J. \& Deady, R. Moral distress reconsidered. Nursing Ethics. 2008; 15(2): 254-262. PMid:18272615 http://dx.doi.org/10.1177/0969733007086023

[8] Fourie, C. Moral distress and moral conflict in clinical ethics. Bioethics. 2013; 28 (3): n/a-n/a (online). http://doi.wiley.com/10.1111/bioe.12064

[9] Hamric, A. Empirical research on moral distress: Issues, challenges, and opportunities. HEC Forum. 2012 ; 24 : 39-49. PMid:22476738 http://dx.doi.org/10.1007/s10730-012-9177-x

[10] Corley, M. Moral distress in critical care nurses. American Journal of Critics Care. 1995; 4: 280-85. PMid:7663591

[11] Stanley, Mary Jo C. Determining the meaning of student nurses' clinical experiences with diverse populations (Unpublished doctoral dissertation). University of Northern Colorado, Greeley. 2012.

[12] Denzin, N. K. Interpretive interactionism, (2nd Ed.). Thousand Oaks, CA: Sage. 2001. PMid:11381132

[13] Yin, R. Case study research design and methods (3rd Ed.). Thousand Oaks: Sage Publications. 2003.

[14] Stanley, Mary Jo C. Examining Student Nurses' Perceptions of Diverse Populations: Are Student Nurses Prepared to Care for Culturally Diverse Patients? Journal of Nursing Education and Practice. 2014; 4(7): 1-8.

[15] Elpern, E. Moral distress of staff nurses in a medical intensive care unit. American Journal of Critical Care. 2005; 14: 523-30. PMid:16249589

[16] Brazil, K., Kassalainen, S., Ploeg, J. \& Marshall, D. Moral distress experienced by health care professionals who provide home-based palliative care. Social Science and Medicine. 2010; 71: 1687-1691. PMid:20832923 http://dx.doi.org/10.1016/j.socscimed.2010.07.032

[17] Epstein, E. \& Delgato, S. Understanding and addressing moral distress. The Online Journal of Issues in Nursing. 2010; 15(3): Manuscript 1. 